\title{
DÉBAT SUR LE FRONT POLAIRE
}

\author{
Jean-Jacques Thillet \\ Météo-France \\ CDM de l'Isère \\ 1441, rue de la Piscine \\ Domaine universitaire \\ 38406 Saint-Martin d'Hères Cedex \\ et Alain Joly \\ Météo-France \\ Centre national de recherches météorologiques \\ 42, avenue Gustave-Coriolis \\ 31057 Toulouse Cedex
}

Le numéro de février 1995 de la revue La Recherche a publié un article d'Alain Joly intitulé : «Le front polaire : un concept dépassé... qui a la vie dure ». À la suite de cette parution, Jean-Jacques Thillet a adressé à Alain Joly, le 10 mars 1995, une lettre intitulée "Défense et illustration du front polaire ", lettre à laquelle ce dernier a répondu le 16 mai 1995.

II ne s'agit pas d'une querelle entre anciens et modernes, mais d'un débat dans lequel la complémentarité des compétences des auteurs s'allie à un souci commun de garder vivante une météorologie des concepts, en dépit des évolutions techniques et informatiques.

Une partie des illustrations parues dans La Recherche sont reproduites ici. Mais le lecteur est évidemment invité à se reporter à l'article qui est à l'origine de cet échange de correspondance. (Ndlr)

Débat sur l'existence du front polaire entre Jean-Jacques Thillet, prévisionniste formé à la théorie norvégienne des perturbations, et Alain Joly, chercheur en météorologie dynamique.

ABSTRACT

A discussion on the existence of the polar front between Jean-Jacques Thillet, an experienced forecaster trained on the Norwegian theory of weather disturbances, and Alain Joly, a researcher in dynamical meteorology.

DÉFENSE

ET ILLUSTRATION DU FRONT POLAIRE par Jean-Jacques Thillet
J'ai lu avec beaucoup d'attention votre article dans La Recherche de février. Je l'ai abordé avec une certaine inquiétude, pensant qu'il allait réduire en miettes non seulement les acquisitions de ma formation initiale en météorologie, mais aussi les suppléments que ma réflexion personnelle avait ajoutés, à partir de mon expérience de prévisionniste. En définitive, je suis rassuré, en accord avec presque tout, sauf... le titre et la conclusion.

J'exprime d'abord ma satisfaction d'avoir enfin pu prendre connaissance des récents développements sur la théorie des tempêtes et, plus généralement, sur l'image que l'on se fait maintenant de la structure et de la vie de la troposphère. Je sais bien qu'il existe des ouvrages beaucoup plus savants, mais il faut avoir le temps de les lire, de les assimiler, ce qui n'est d'ailleurs pas facile, quand ce n'est pas tout à fait impossible, vu le formalisme mathématique employé. Évidemment, l'article en cause est un texte de vulgarisation - haut de gamme tout de même - avec ses contraintes de simplification, sans doute frustrantes pour l'expert, mais parfaites pour le praticien standard. 
Je ne suis pas d'accord avec le commentaire de la figure 1. Ce n'est pas parce qu'il y a rupture flagrante dans la distribution nuageuse qu'elle s'accompagne automatiquement d'une disparition de la discontinuité thermique frontale. Votre explication ne peut convaincre que le profane. On peut fort bien imaginer entre les deux perturbations une poche froide aux limites méridionales inactives mais bien réelles ; un cas ordinaire.

La reconstitution de l'histoire de la découverte du schéma de la perturbation extratropicale par l'équipe de Bergen (figure 2) est fort intéressante. Cependant, je trouve que le ton est un peu injuste pour ces physiciens norvégiens. La réussite de la méthode est présentée comme un splendide coup de publicité, un succès pas vraiment mérité. Cette « théorie » n'est peut-être pas géniale pour un scientifique de haute volée et exigeant, elle n'atteint certes pas la sophistication mathématique de la théorie de la relativité universelle, mais quelle découverte pratique pour la majorité des météorologistes du monde entier, quel fantastique outil de communication avec les usagers les plus exigeants (aviation, marine...) comme avec les moins avertis (grand public) ! Et cela dure depuis plus de 70 ans ! Récemment, un croquis dans un grand quotidien national expliquait les inondations exceptionnelles de janvier par une série d'ondulations perturbées sur le «front polaire » étiré des Bermudes à l'Europe centrale. Sobre, simple, efficace, et, à mon avis, parfaitement justifié. Si l'invention était française, elle jouirait sans doute chez nous d'un prestige plus éclatant. Question d'époque vraisemblablement, car les Viaut, les Pône (et son remarquable cours sur les masses d'air), et bien d'autres anciens météorologistes renommés ont, pour ce que j'en sais, complètement adhéré au concept et l'ont même peaufiné.

J'ai cru comprendre que la découverte du courant-jet fut un pavé dans la mare de Bjerknes et de ses disciples parce qu'il ne révélait pas les gradients intenses qui auraient dû accompagner le front polaire. Les discontinuités brutales de température, d'humidité..., existent pourtant bel et bien. L'effet du sol (convectif, radiatif...) accentue sans doute les contrastes, mais l'examen des enregistrements d'altitude (quasiment en air libre) met en évidence des fluctuations rapides et très

Figure 1 - La tempête du 10 janvier 1993.

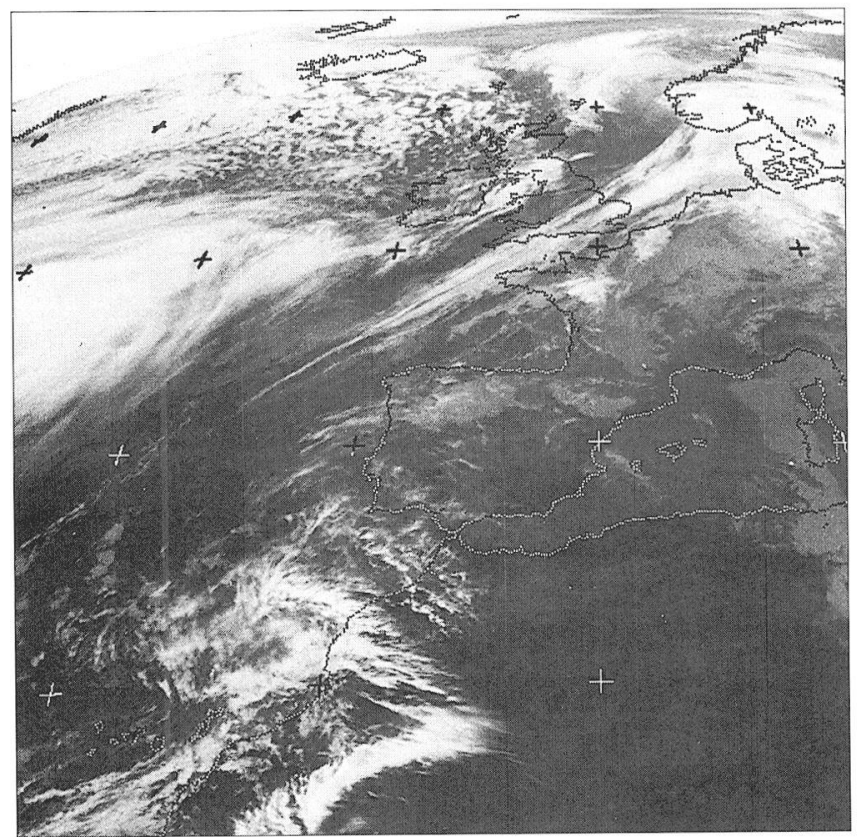

a) Image du 9 janvier 1993 à 12 h UTC. Canal infrarouge.

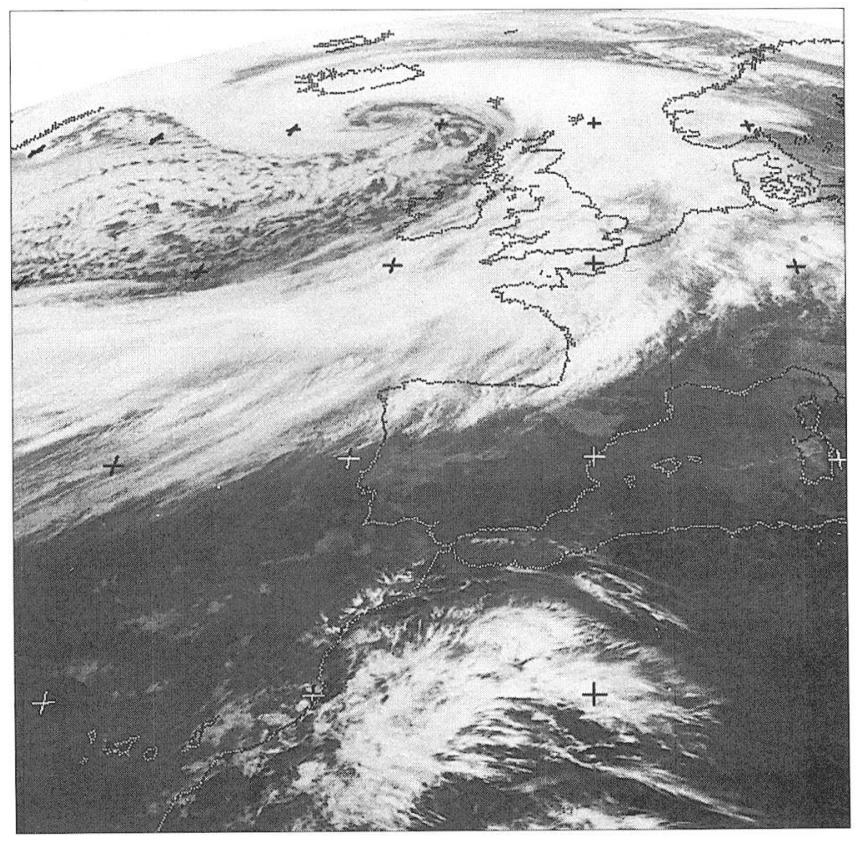

(b) Image du 10 janvier 1993 à 12 h UTC. Canal infrarouge. Les tempêtes comme celle du 10 janvier 1993, illustrée ici par des images des satellites géostationnaires Météosat, figurent au premier rang des sources du " mauvais temps " sous nos latitudes. Souvent, on les explique en termes de conflit entre air tropical et air polaire. La ligne de contact entre ces masses d'air très contrastées serait le «front polaire». Les fortes différences entre ces deux airs le rendraient instable et cela entraînerait la formation de tempêtes. Cette conception remonte à 1920 et reste très présente. Selon ce point de vue, les tempêtes comme celle entraînerait la formation de tempêtes. Cette conception remonte à 1920 et reste très présente. Selon ce point de vue, les temper d'œil - enfilées sur le qui apparait en (b) à son stade de mare le l'image (a) la masse nuageuse informe qui va devenir la belle spirale au centre de l'image (b): elle se présente manifestement comme un objet totalement indépendant de la dépression qui la précède sur l'Angleterre (le front froid de cette dernière, la fine bande nuageuse qui s'étend vers le sud-ouest, ne rejoint pas le paquet précurseur de la tempête du 10 janvier). Le front polaire, en effet, n'existe pas. Photos: Météo-France, CMS Lannion. Légende tirée de l'article de Joly (1995). 
marquées (les chutes de $7{ }^{\circ} \mathrm{C}$ ou plus en quelques minutes ne sont pas rares en montagne). Le courant-jet, si j'ai bien compris, résulte de l'accumulation de faibles « épaisseurs » dans l'air froid, d' « épaisseurs » plus conséquentes dans l'air chaud. Il ne détruit pas le concept du front polaire, il le complète. Il est bien normal d'ailleurs que la théorie progresse avec les moyens d'investigation (il est tout de même étonnant qu'elle ait si bien vieilli, confortée par l'évolution des outils de mesure, de la radiosonde au satellite).

J'ai relevé la phrase suivante : «Cette transition se retrouve à tous les niveaux entre la surface et l'altitude du maximum du jet : elle est profonde et s'étend presque tout autour de la Terre ». Je trouve qu'elle n'est vraiment pas loin de la définition classique du front polaire.

Il est vrai que l'on sait maintenant qu'il existe des fronts froids surplombants, étrangers au schéma classique. Je le savais déjà par expérience, il y a vingt ans (front freiné au sol en pénétrant dans un champ de pression mou et progressant plus vite en altitude, par exemple). Important, mais ce n'est qu'une variante qui ne remet pas en cause le schéma de base, forcément un peu caricatural.

La différence fondamentale entre la théorie norvégienne et l'approche actuelle semble résider dans la nature de l'impulsion qui amorce la déformation du front polaire. Une instabilité thermique mystérieuse présidait auparavant à la naissance des perturbations ; on fait appel désormais à une influence intense externe, mécanique : les tourbillons migrateurs. Je note au pas-

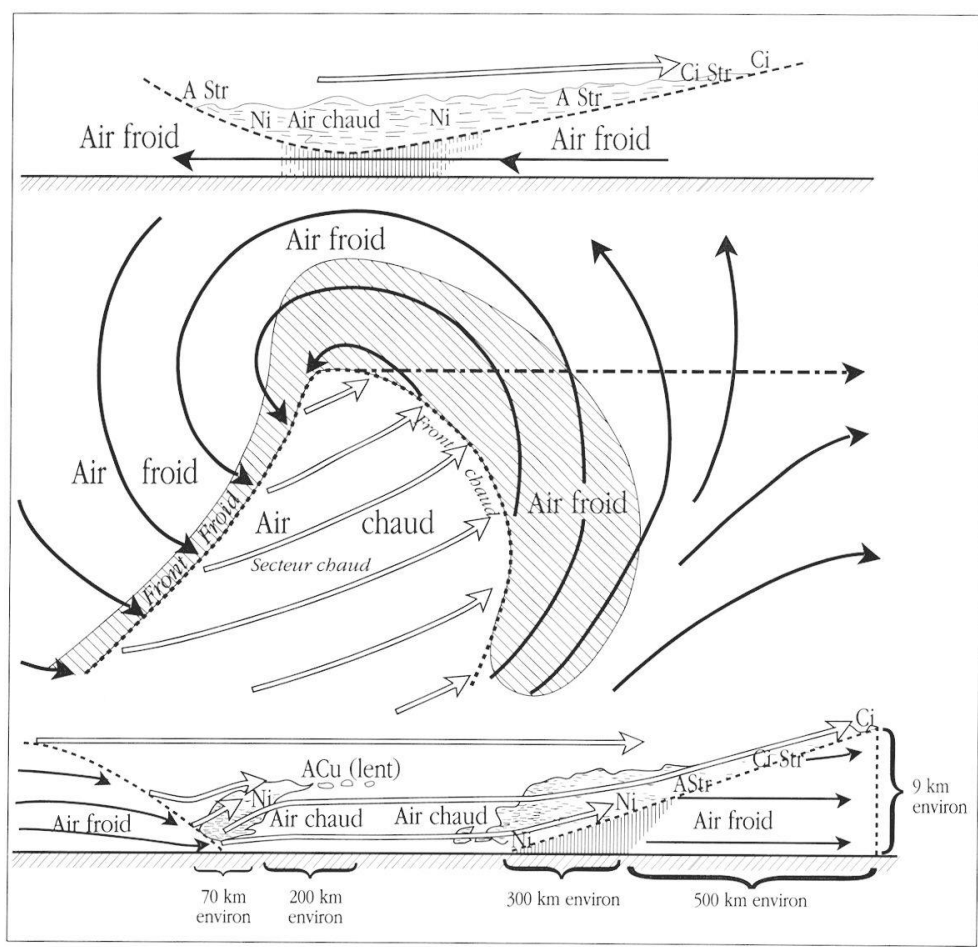

Figure 2 - Schéma de perturbation selon l'école norvégienne. La partie supérieure de la figure est une coupe verticale de la dépression par un plan passant au nord de son centre et la partie inférieure est une coupe verticale par un plan passant au sud (Ndlr). Figure tirée de Bjerknes et Solberg (1923) sage que le hasard intervient encore dans la répartition efficace ou inhibitrice des tourbillons de surface et d'altitude. A-t-on le droit d'évoquer le hasard? Je ne sais pas. Stérile querelle de mots sans doute...

En 1985, j'ai exposé dans un document interne ma façon de concevoir la genèse des perturbations. Cela n'a intéressé personne, et pourtant il me semble que l'idée n'était pas mauvaise (d'ailleurs je l'ai exprimée par écrit seulement à cette date, mais je l'avais en tête depuis une quinzaine d'années). Le tourbillon moteur (peutêtre le «précurseur » de l'article), j'en concevais l'origine dans des gouttes polaires se détachant par saccades de la calotte froide (tels des icebergs de l'inlandsis) et qui, au départ, anticycloniques dans les deux ou trois premiers kilomètres, dépressionnaires subsidentes au-dessus, se transformaient en dépressions convergentes sous l'effet de la convection aux latitudes tempérées, en survolant des surfaces maritimes adoucies. Ces pôles froids tourbillonnants, accompagnés de jets à leur périphérie chaude (jets qu'ils provoquent mais sur lesquels ils appuient ensuite leur mouvement dans un effet rétroactif), étaient très repérables au niveau de la tropopause. D'où l'intérêt aigu que j'accordais alors à la carte «Tropopause et vents maximaux » de l'époque (ce qui ne manquait pas de susciter autour de moi des sourires sceptiques...). Les cartes d'analyse et de prévision du tourbillon absolu, qui arrivent depuis assez peu de temps en CDM, permettent de constater, de manière assez évidente je crois, qu'il existe une concordance remarquable entre les tourbillons absolus ascendants et les cœurs de gouttes froides, là où se situe la dynamique qui excite le front polaire. Conception simple, simpliste, «recette »? En tout cas, méthode fort utile pour réussir des prévisions à une époque où les modèles n'existaient pas.

«Il faut retenir ici que les fronts résultent de la croissance de la tempête, ils ne la précèdent pas ». Je suis presque d'accord, à la nuance près que j'estime que le front polaire existe tout le temps. Je pense qu'il faut distinguer le front polaire inactif, aux isothermes distendues, du front polaire actif (front chaud, front froid, 
occlusion), aux isothermes rapprochées par insertion en force de l'air chaud dans l'air froid. Cette poussée crée, par ascendance, une dépression centrée au voisinage du sommet de l'onde, l'ascendance étant elle-même d'autant plus forte (et le creusement plus actif) que l'attraction du tourbillon moteur est puissante, le contraste entre air froid et air chaud accentué, le dégagement de la chaleur latente intense, l'instabilité de l'air tropical accusée. Pour le reste, j'accepte volontiers l'existence de tourbillons dissociés, d'altitude et de surface, résidus de dépressions en voie d'extinction, ou prémices de nouvelles, et dont la disposition relative détermine ou bien une synergie (couplage) ou bien une neutralisation réciproque.

La conclusion m'inquiète un peu, en tant que « vieux » prévisionniste. Je ne veux pas développer ici mes arguments (je l'ai fait en d'autres circonstances), mais je dois dire, en dehors du fait que je la conteste, qu'elle ne peut guère motiver les météorologistes qui s'appliquent, trop rarement aujourd'hui, à tracer des fronts devenus bien archaïques. C'est regrettable pour la compréhension de la vie de l'atmosphère, pour la prévision immédiate, pour nos relations avec l'extérieur: comment nos usagers non initiés, le plus grand nombre, comprendraient-ils une carte de tourbillons?

Ces réflexions sont celles d'un ancien praticien, toujours passionné ; elles n'ont aucune intention polémique. Je suis attaché - par la culture, par la réflexion (j'ai essayé de le prouver) - à une représentation la plus accessible possible, en interne et en externe. Il me semble que la conception traditionnelle et la nouvelle ne s'opposent pas vraiment, mais peuvent au contraire plutôt se marier harmonieusement en se renforçant mutuellement.

\section{RÉPONSE Votre lettre du 10 mars dernier ne restera pas sans réponse. Mon retard par Alain Joly $\begin{aligned} & \text { s'explique par la priorité du projet Fastex, évoqué à la fin de } \\ & \text { Recherche, et j'espère que vous ne m'en tiendrez pas trop rigueur. }\end{aligned}$}

Je vous remercie d'avoir lu l'article avec attention et d'avoir tenté de faire la part des choses. Sur cette question, je redoute un peu les réactions épidermiques. Je m'en voudrais beaucoup de blesser, au travers d'une lecture superficielle, des prévisionnistes expérimentés ou ayant exercé autrefois. Rien ne saurait être plus éloigné de mes intentions.

Votre lettre pose avant tout la question de fond suivante : pouvons-nous continuer de faire progresser la prévision du temps en conservant la terminologie de front polaire?

Vous répondez par l'affirmative et vous mélangez, à l'appui de votre plaidoyer, des arguments d'expérience, de technique et de nature historique. De mon côté, je pense que les concepts dont nous parlons ici (courant-jets, fronts, etc.) possèdent un réel intérêt pratique dans l'expression d'une prévision plus riche et plus réfléchie. Ces concepts ne sont pas pour moi un moyen de vulgarisation ou de communication avec le grand public, ce sont nos outils de travail. À ce titre, on devrait les entretenir, les affûter. Je constate que leur usage est, au contraire, devenu un carcan inhibiteur.

Voyons ces arguments. Le front polaire possède une immense importance historique. Tout à fait d'accord avec vous. L'école norvégienne fonde les premiers vrais services publics de prévision, sortant ce travail de l'amateurisme et de l'impuissance chronique. Le concept de front polaire autorise une certaine prise sur la moyenne échéance et permet d'accompagner de manière utile les débuts de l'aviation long courrier. L'article dit tout cela sans ironie aucune.

Toutefois, le fait pour une idée de permettre un progrès, même immense, n'interdit pas qu'elle forme le socle de nouvelles propositions qui la dépasseront par la suite. Les concepts développés autour de la notion de front polaire constituent une étape nécessaire et essentielle de notre compréhension actuelle de la troposphère des latitudes tempérées ; personne ne le conteste. Les idées actuelles émergent des idées norvégiennes. Vous exprimez ces vues, mais votre lettre 


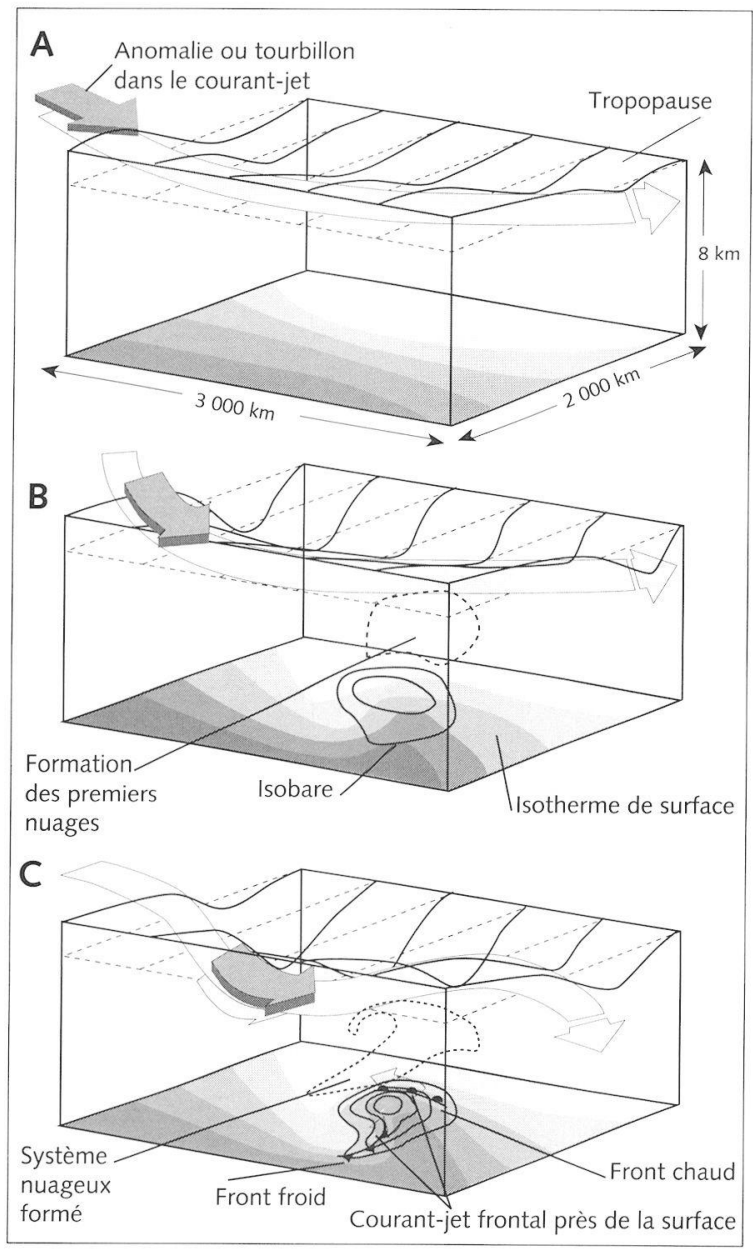

Figure 3 - La formation d'une tempête est ici schématisée selon la théorie actuelle. Le scénario illustré conduit aux tempêtes les plus soudaines et les plus intenses, comme celle du 15 octobre 1987 ou du 10 janvier 1993 (voir figure 1). L'état initial en A montre le courant-jet avec un précurseur en altitude arrivant en amont, c'est-à-dire un tourbillon que figure la flèche à gauche du dessin. II n'y a pas de fronts. Sous le jet, se situe une région de variation continue, modérée, de la température. Ce tourbillon peut provenir de ce qui reste d'une tempête précédente disparue loin en amont. La phase de début de creusement de la dépression de surface est représentée en B. Arrivant par l'ouest, le tourbillon d'altitude se transforme en tempête quand il peut induire ou rattraper un début de dépression en surface Une nouvelle dépression (marquée sur la figure par les isobares) apparaît un peu en aval du précurseur d'altitude. Les mouvements horizontaux et verticaux qui apparaissent alors convertissent en vent l'énergie thermique contenue dans le contraste de température modéré associé au jet (les isothermes se sont rapprochées). Le vent ainsi créé permet aux masses d'air de se déplacer. Les premiers nuages apparaissent en paquet au-dessus de la jeune dépression. Le jet se déforme un peu (effet de l'amplification du tourbillon en altitude). La phase de maturité est illustrée en C. Le jet d'altitude est aussi très déformé, ainsi que la tropopause. Tourbillon d'altitude et dépression de surface se renforcent mutuellement. Ce renforcement se traduit ici par le doublement de la flèche en altitude. En surface, la pression baisse davantage et le vent souffle plus fort partout. En certains endroits, il se renforce vraiment beaucoup : de véritables jets apparaissent près du sol. En ces mêmes endroits, les isothermes considérablement resserrées permettent alors de parler de fronts. Entre surface et altitude, la spirale nuageuse caractéristique s'est constituée. Si la configuration C se maintient et que le jet s'étend toujours vers l'est, le développement de la tempête continue. Sinon, il se ralentit ou s'arrête.

Figure et légende tirées de l'article de Joly (1995)

montre que vous en sous-estimez la profondeur : c'est le même Jakob Bjerknes qui a avancé le schéma de 1920, puis l'a remis en question. Pourquoi admirez-vous moins le Bjerknes critiquant le front polaire que le Bjerknes le proposant comme une hypothèse audacieuse?

En sciences, dépasser une idée, la remplacer par de nouvelles, ne retire rien à l'importance de l'idée d'origine dans son contexte historique, mais en révèle la fécondité.

Voyons les arguments techniques. Nous avons d'abord un point essentiel à clarifier. Vous écrivez : «Les discontinuités brutales de température, d'humidité... existent pourtant bel et bien ». Deux interprétations sont possibles. Voulez-vous dire par là que l'article nie le concept de front météorologique ? Ce n'est pas le cas, puisque la dernière colonne de la page 134 de 1'article de La Recherche replace les fronts dans leur contexte et que le troisième schéma de la dernière figure (voir figure 3 ) fait de même graphiquement. Le concept de front demeure un outil conceptuel central dans l'analyse météorologique.

La seconde interprétation soulève la question clé de la définition pratique du front : vous semblez en effet confondre les fronts et le front polaire. Observer ici et là de fortes discontinuités ne signifie pas vraiment que ladite discontinuité fait le tour de la Terre et encore moins que ses ondulations créent des dépressions (raisonnement bancal du genre «l'existence du front polaire prouve l'existence de son instabilité »). Cela prouve seulement que le concept de front correspond à une réalité, ni plus, ni moins.

Cette confusion entre front et front polaire, je la rencontre souvent. À cause d'elle, on ne sait plus très bien quelle réalité recouvre ce terme de front. Ce problème existe aussi graphiquement sur le pré-iso ${ }^{(1)}$ : on ne sait plus très bien si celui qui dessine un front représente le front polaire qui passait par là, une bande nuageuse, une zone de précipitations (intenses, pas intenses ?), une ligne de grains ou... un front au sens dynamique du terme, le seul qui devrait être admissible.

À l'origine de cette confusion, on trouve une définition rendue trop élastique par l'application du même concept à des réalités très différentes. L'existence de cette confusion constitue le principal argument pour introduire des termes distincts, bien définis, pour les structures aujourd'hui comprises et utiles à la synthèse, et pour limiter l'usage de l'expression front polaire à son sens original (donc

(1) Ndlr : Le pré-iso (ou carte pré-iso) est une carte de prévision en surface comportant le tracé des isobares et celui des fronts. Cette carte, tracée par les prévisionnistes à partir des résultats des modèles de prévision numérique, est un document technique essentiel qui synthétise, sous une forme graphique, la prévision synoptique. La présentation du pré-iso s'appuie, encore actuellement, sur le symbolisme norvégien. 


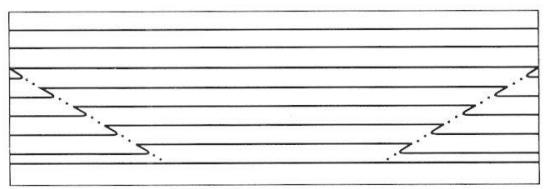

Figure 4 - Schéma de la structure thermique des surfaces frontales selon la conception norvégienne.

Coupe verticale effectuée au sud du centre d'une dépression en cours de développement. Isothermes en trait continu et lignes de discontinuité en pointillés. Figure tirée de Bjerknes et Solberg (1923). peu pertinent dans la pratique actuelle). Il est essentiel pour notre travail de recourir à des définitions univoques des concepts que nous utilisons dans nos bulletins, dans nos synthèses graphiques. À l'acquisition de ces définitions s'associe une image mentale qui doit être la même pour tous.

Qu'évoque pour vous l'expression « front polaire »? Si vous appelez front polaire une zone barocline large et modérée, sans cisaillement horizontal de vent marqué en surface, mais associée à des vents forts en altitude, incontournable règle du vent thermique ${ }^{(1)}$, structure que je préfère appeler courant-jet, alors nous sommes d'accord. Du fait de la succession de zones d'accélération et de décélération, le courant-jet induit des mouvements verticaux que des nuages peuvent matérialiser. Dans ce sens, on peut employer les deux terminologies.

En revanche, si vous pensez aussitôt à la figure 4 tirée de Bjerknes et Solberg (1923), alors nous ne pouvons réconcilier nos points de vue. En effet, ces structures verticales n'existent pas. Je ne pense pas ici aux structures verticales que l'on peut inventer entre deux radiosondages espacés de 500 kilomètres. Je pense à la véritable structure observée par des sondages rapprochés et par des radars Doppler. C'est sans appel. On sait observer la structure verticale d'un front et, de plus, on sait en retrouver les principales caractéristiques par le calcul. On sait ainsi qu'il faut réunir, pour produire les quasi-discontinuités parfois observées, un champ de déformation géostrophique intense, mais aussi une action de la convergence agéostrophique que seules les ascendances au cour des dépressions peuvent entretenir.

Il m'apparaît ainsi très utile, de manière à disposer de concepts opérationnels univoques, de distinguer, d'une part, le ou les courants-jets (zones baroclines larges et modérées, très longues) et, d'autre part, les fronts (zones baroclines étroites, intenses, de longueur limitée, soulignées par un gradient de température autant que par des cisaillements de vent près de la surface.

Comme il existe un risque de confusion, que vous illustrez, entre les images originale et évoluée du front polaire, il vaut mieux ne plus utiliser cette expression. C'est le seul moyen de se comprendre sans équivoque.

Vous n'êtes toujours pas convaincu? Pourtant, c'est très simple. Si je dis « un chien s'approche », l'image en général amicale ou neutre d'un chien se forme dans votre esprit. En revanche, si la même phrase appelle pour vous l'image d'un loup, avec toute la charge émotionnelle que véhicule ce mot, je ne peux plus comprendre vos réactions. De plus, vous m'interdisez ainsi de marquer une gradation dans le risque en disant plus tard «voilà un loup », puisque vous êtes déjà effrayé. Il existe bien un lien biologique très fort entre chien et loup (il s'agit quasiment de la même espèce). Contestez-vous pourtant qu'il soit utile de distinguer les deux animaux ? Il existe de même un lien de parenté dynamique, que vous connaissez, entre courant-jet et front, mais il est utile de distinguer les deux, pour la clarté de la prévision. La chose me semble plus facile entre courant-jet et front qu'entre front polaire et front.

Le problème n'est pas seulement sémantique. Pour enrichir le pré-iso, le rendre plus parlant, il faudrait représenter, parfois au même endroit, le courantjet (héritier du front polaire, donc) et des fronts. La réalité observée est comme cela et nous comprenons aujourd'hui plutôt bien pourquoi elle est ainsi. Dans le cadre de la théorie norvégienne, cette superposition est chose impossible, les fronts étant conçus comme des morceaux du front polaire. Pour avancer, il faut donc disposer de deux concepts distincts.

Résumons donc les avantages et les inconvénients du front polaire. Quels sont les attributs du front polaire que les travaux des Bjerknes eux-mêmes et de leurs successeurs ont complètement remis en question?

- Le front polaire est une discontinuité (plus ou moins active, plus ou moins «épaisse ») permanente, circumterrestre. Faux : au mieux, on a le système de courants-jets qui, en particulier, ne correspond à aucune discontinuité du vent (contrairement au schéma de Margules utilisé pour le front polaire). De plus, les gradients de température sont modérés.

(1) Ndlr : Le vent varie suivant la verticale en fonction du gradient horizontal de température ; c'est la relation du vent thermique. 
- Les dépressions naissent de l'instabilité du front polaire. Faux : ce mécanisme conduit à de l'instabilité barotrope, qui ignore l'énergie thermique. Or, les dépressions convertissent l'énergie thermique pour donner du vent : cela, les Bjerknes le savaient bien avant d'inventer le front polaire.

- Le front polaire est créé par les anticyclones et les dépressions permanents que nous révèle la climatologie. Faux : tout d'abord, ces structures sont des artefacts des opérations de moyenne, et leur variabilité est en fait considérable (le champ de déformation induit de la sorte est tout sauf permanent) ; ensuite, la déformation créée manque d'intensité.

Quels sont les attributs du front polaire qui préparaient bien notre compréhension de l'atmosphère aux latitudes moyennes et que l'on pourrait conserver, moyennant une nouvelle définition du concept?

- Le front polaire est la zone privilégiée où se développent les dépressions. Vrai, mais tout le contenu de cette idée est différent : la structure du front polaire est tout autre (courant-jet), le mécanisme d'amplification des dépressions est barocline, l'origine des dépressions est différente. En particulier, la série de dessins montrant un front polaire ondulant sous l'effet de sa propre instabilité ne peut que donner une image fausse de la cyclogenèse. Or, ce sont ces dessins que l'on associe le plus souvent au front polaire.

Il me semble que ce bilan justifie que l'on renonce à utiliser en météorologie opérationnelle un terme si peu lié à la réalité et qui appelle des images si confuses. Cela n'enlève rien à son importance historique, ni à son caractère précurseur.

Pour compléter ma réponse, je vais revenir sur les autres aspects de votre lettre point par point.

Ainsi, dans le second paragraphe, vous semblez considérer qu'une justification mathématique des idées vous interdit l'accès à ces dernières. Pourtant, si les questions que vous évoquez dans votre courrier vous semblent vraiment importantes, vous n'échapperez pas à un minimum de justification physique et mathématique. On s'égare dès que l'on essaie de réduire à des mots et à des concepts flous la mécanique d'un fluide stratifié quasi eulérien en rotation.

Je me permets de relever ce point dans la mesure où il constitue l'un des aspects abordés par l'article de La Recherche. Nous constatons que la théorie physique et mathématique du front polaire ne donne pas de résultats qui valident le concept de la cyclogenèse par ondulation du front polaire. C'est ainsi. Dans le sens où, en physique, une théorie prévoit des grandeurs observables au terme d'une démonstration sous des hypothèses conformes aux grands principes (conservation de la masse, de l'énergie, etc.), il n'existe pas de théorie du front polaire. Cela ne se devine pas hors du calcul car, incontestablement, le modèle norvégien séduit l'intuition.

Je passe au paragraphe suivant. Là, vous marquez un bon point : je vous accorde que le raccourci de l'image (figure 1) et de sa légende ne constituent pas une démonstration. En réalité, les principaux faits contredisant la version norvégienne de l'origine des dépressions figurent dans le corps de l'article. Par ailleurs, les anomalies au comportement norvégien orthodoxe sont multiples. Un exemple classique concerne les trains de perturbations, comme cet ensemble de trois dépressions qui se trouvaient le 15 octobre 1987 sur l'Atlantique. La plus creuse était la plus à l'est. Dans une théorie d'instabilité purement frontale, c'est elle qui aurait dû continuer de s'amplifier le plus. Comme vous le savez sûrement, c'est en fait celle située le plus à l'ouest qui s'est transformée en tempête majeure. Cet argument est trop complexe pour être utilisé dans La Recherche. La difficulté, mal résolue dans l'article, est de trouver un contre-exemple qui tienne en une image et une phrase.

Je suis surpris par ce qui vient ensuite, à savoir vos remarques sur l'histoire des sciences. J'ai écrit : «Plusieurs raisons expliquent ce succès. Il faut le dire, ces concepts comblaient avant tout une forte attente des météorologistes. Ils apportaient une synthèse un peu plus profonde que, par exemple, le système nuageux français, et surtout beaucoup plus claire, plus simple à expliquer. Plus important encore, ces idées s'accompagnaient de tout un ensemble de conséquences pratiques fort appréciées, qui constituaient un réel progrès ». Ce paragraphe indique tout ce qu'a apporté la méthode norvégienne, et je ne vois pas ce que le ton a de spécial. Peut-être cela ne se voit-il pas assez, mais je voue une immense admiration 
à Jakob Bjerknes qui a su mener une révolution des idées puis qui, loin de se satisfaire du résultat, en a remis les bases en question.

Je signale en effet le poids de la politique de communication dans la rapidité de la diffusion du modèle norvégien. Je crois, d'une part, qu'il s'agit d'un aspect nouveau, peu connu ; d'autre part, il faut comprendre qu'en 1920, rien n'était joué : il n'existait pas de déterminisme a priori en faveur de l'approche norvégienne. Un colonel Delcambre plus agressif et plus déterminé que Vilhelm Bjerknes aurait très bien pu imposer au monde météorologique le système nuageux et surtout la méthode des noyaux. Il est significatif que vous citiez Viaut mais pas Wehrlé, Schereschewsky ou Bureau, les inventeurs de la «méthode française », seule méthode vraiment conçue pour faire des prévisions (la méthode norvégienne est surtout une méthode d'analyse), méthode inventée et éprouvée pendant la Grande Guerre dans un des pays qui vient de la gagner. C'est significatif de ce qu'il faut bien appeler le reniement par les Français d'une grosse part de leur contribution à l'évolution de la météorologie.

Pour en rester à l'école française, Robert Pône joue un autre rôle, ayant imaginé la vision la plus complète possible en termes de masses d'air. Cette approche présente de nombreux aspects positifs, utiles et avantageux. Elle est quand même biaisée vers la thermodynamique. Comme vous le dites, il s'agit en effet d'une généralisation éclatante de la vision norvégienne, inspirée de travaux de Bergeron.

Je ne vous suis pas du tout quand vous trouvez remarquable que l'on serve le front polaire comme «explication » des inondations de janvier 1995 dans le Nord de la France. Il s'agit à mes yeux d'une démission devant notre rôle pédagogique. Je vous signale qu'un journal régional (de Picardie ou du Nord) n'a pas hésité à se tourner vers les chercheurs de l'École normale supérieure (Robert Vautard, du Laboratoire de météorologie dynamique) pour obtenir des explications plus modernes. Le résultat est un peu piquant (trop d'effet papillon, des simplifications osées du journaliste), mais pas moins clair que le très usé front polaire ; il introduit la très importante notion de régime de temps, bien plus pertinente ici que le front polaire. En effet, la question de ces inondations n'est pas de savoir d'où vient l'eau, mais pourquoi le jet, ou le front polaire si vous préférez, n'a pas, pendant aussi longtemps, suivi son orientation habituelle vers le nord-ouest. Que disent les Norvégiens là-dessus ? Rien.

J'avoue ne pas comprendre ce qu'est une «accumulation d'épaisseurs », ni comment le jet peut en "résulter». Courant-jet et gradient thermique horizontal s'accompagnent mutuellement dès lors que l'équilibre du vent thermique s'impose : aucun des deux termes ne constitue une cause de l'autre. Le jet résulte des différences d'éclairement de la Terre par le Soleil et surtout de la rotation de la Terre.

Il existe, à toutes les échelles, une foule de phénomènes étrangers au schéma classique : des fronts de toutes les formes horizontales et verticales, des dépressions qui se forment loin d'un front, d'autres qui persistent sans baroclinie, etc. J'en profite pour insister sur une phrase de l'article : le schéma conceptuel de cyclogenèse proposé n'est pas unique et ne prétend pas à une quelconque universalité. Il s'agit seulement du mécanisme à l'origine des tempêtes soudaines, pas de toutes les dépressions.

Je continue d'avancer. L'instabilité du front polaire n'est ni thermique ni mystérieuse : on savait presque tout depuis 1950, on sait tout depuis 1968. Vous mettezlà le doigt sur une autre limite du discours type des tenants du front polaire : d'un côté on essaie de faire croire que «c'est bien connu », de l'autre très peu de gens connaissent vraiment le contenu du travail des Norvégiens. Le schéma norvégien était omniprésent dans ma formation initiale à l'analyse et à la prévision, mais pourtant, personne ne m'en a jamais fait une présentation formelle. Pourquoi ?

J'ai employé en effet l'expression « rencontre de hasard» et ce mot en fait sursauter plus d'un. Si l'on considère la population des tourbillons ou des anomalies neutres qui se propagent paisiblement dans l'atmosphère, on voit que certains se développent en dépressions, d'autres pas, selon la nature des structures situées sur leur trajectoire. J'emploie, peut-être à tort, ce mot de hasard pour illustrer ce fait un peu statistique. Cela ne veut absolument pas dire qu'un événement précis est imprévisible. L'identification et le suivi des anomalies en question assurent, si le système d'observation le permet, une excellente prévisibilité des cyclogenèses. 
Nous en venons à votre proposition sur l'origine des ondulations du front polaire. Par certains côtés, vous partagez la vision actuelle en accordant, comme René Mayençon, l'importance qu'il faut à la situation d'altitude (approche qui n'est pas, vous le savez, conforme à l'orthodoxie norvégienne, manière 1920). C'est, selon mes lectures, la bonne direction à prendre. René Mayençon connaît bien le mécanisme mis en avant par la figure 3 pour l'avoir « réinventé » indépendamment des écoles scientifiques, ce qui lui a permis de réaliser ses prévisions les plus spectaculaires.

Par d'autres, vous vous engagez sur des voies qui conduisent tout droit aux anticyclones mobiles polaires de Marcel Leroux, objets dont l'identité dynamique reste à définir et à valider. L'image des icebergs est élégante, mais je ne vois pas du tout ce qui peut rapprocher ces phénomènes (par exemple, l'un est saisonnier, l'autre pas ou peu). Les objections à ces idées ne manquent pas. Pourquoi la calotte froide " expulserait-elle des masses d'air », ou des gouttes froides se «détacheraient-elles par saccades »? C'est aussi mystérieux que « la langue d'air chaud qui s'enfonce comme un coin dans une masse d'air froid », vous ne trouvez pas ? Si certains apprécient le phénomène du point de vue de l'air chaud, vous préférez, avec M. Leroux, vous placer du point de vue de l'air froid. Tous ces points de vue se défendent et recouvrent tel ou tel aspect de la réalité. Leur intérêt respectif dépend de l'usage que l'on veut en faire.

Pour la prévision, c'est-à-dire l'anticipation la plus avancée possible, l'identification de précurseurs (qui sont, du point de vue dynamique, des paquets d'ondes de Rossby se propageant) semble intéressante, car les précurseurs existent avant que, amorçant une interaction barocline, ils ne permettent à l'air froid (ou à l'air chaud) de bouger. En d'autres termes, ce mouvement de l'air froid qui attire votre attention correspond à un phénomène déjà enclenché et non à un phénomène nouveau.

D’une manière générale, permettez-moi une remarque. Vous vous exprimez beaucoup en termes de température et de densité, donc de thermodynamique statique. Ce faisant, vous risquez de perdre de vue l'essentiel, la dynamique. Les aspects statiques sont du second ordre pour les mouvements qui nous intéressent. Les propriétés du champ de vent géostrophique et le maintien de l'équilibre du vent thermique sont les facteurs les plus importants. Ce sont, en d'autres termes, les conséquences assez peu intuitives (en tout cas pour moi) de la rotation de la Terre. On retrouve une certaine importance de la densité quand on regarde un front à l'échelle du kilomètre et de la microphysique.

Je continue encore un peu. Les isothermes ne sont pas vraiment rapprochées par «l'insertion en force de l'air chaud dans l'air froid». Comme pour les variations de pression, ce sont les mouvements verticaux rétablissant l'équilibre du vent thermique qui font le caractère singulier des fronts (encore la rotation de la Terre). Je suis pris de court par l'image d'un «tourbillon moteur » (je vois bien) « et attracteur » (je vois moins bien). Je dois réfléchir à cette idée.

Me voici arrivé au terme de cette réponse. J'espère ne pas vous avoir trop bousculé : je voudrais vous convaincre qu'il vous faut aller au bout de votre idée (que je partage) selon laquelle la prétendue « nouvelle » conception (Sutcliffe, 1947, Kleinschmidt, 1950 a et b, quand même) s'appuie sur la traditionnelle. La conséquence est que cette vision traditionnelle est maintenant dépassée. Mais cette vision s'est révélée exceptionnellement féconde, ce que l'on ne peut oublier, et elle doit continuer de nous inspirer. Ce n'est pas la même chose que d'en faire un dogme. Le tracé des fronts et des autres structures caractéristiques peut retrouver une seconde jeunesse et redonner beaucoup d'intérêt au travail de prévision : le tout est de rendre toute sa vigueur au concept initial.

BIBLIOGRAPHIE

Bjerknes J. et H. Solberg, 1923 : Les conditions météorologiques de formation de la pluie. L'évolution des cyclones et la circulation atmosphérique d'après la théorie du front polaire. Mémorial n 6 de l'Office national météorologique, Paris, 103 p.

Joly A., 1995 : Le front polaire : un concept dépassé... qui a la vie dure. La Recherche, $26,273,128-135$.

Kleinschmidt E., 1950a : Über Aufbau und Entstehung von Zyklonen I. Meteorol. Rdsch., $3,1,1-6$. 
Kleinschmidt E., 1950b : Über Aufbau und Entstehung von Zyklonen II. Meteorol. Rdsch., 3, 2, 54-61.

Sutcliffe R. C., 1947 : A contribution to the problem of development. Quart. J. Roy. Met. Soc., 73, 370-383.

Friedman R. M., 1989 : Appropriating the weather. Vilhelm Bjerknes and the construction of a modern meteorology. Cornell University Press, Ithaca, États-Unis, 254 p.

Joly A., 1992 : Les tempêtes, les dépressions, comment elles se forment, comment elles évoluent. Idées actuelles sur les perturbations aux latitudes tempérées. Météo-France, Cours et manuels $n^{\circ} 7$, Toulouse, $54 \mathrm{p}$.

Joly A., J.-P. Javelle, F. Lalaurette, S. Malardel, V. Pircher et P. Santurette, 1994 : La conférence de Bergen sur les perturbations extratropicales. La Météorologie $8^{\circ}$ série, 8 , 43-73.

Mayençon R., 1992 : Météorologie marine. Éditions maritimes et d'outre-mer, Rennes, $329 \mathrm{p}$.

Newton C. V. et E. O. Holopainen, 1990 : Extratropical cyclones. The Eric Palmen memorial volume. American Meteorological Society, Boston, États-Unis, 262 p. 